\title{
Pastures planting can ease the conflict between free-range chicken production and the stocking environment
}

\author{
Chunlin $\mathrm{Yu}^{1,2}$, Chaowu Yang ${ }^{1,2 *}$, Huarui $\mathrm{Du}^{1}$, Mohan Qiu ${ }^{1,2}$, Qingyun $\mathrm{Li}^{1}$, Han Peng ${ }^{1}$, Zengrong Zhang ${ }^{1,2}$, Xiaoyan Song ${ }^{1}$, \\ Xia Xiong ${ }^{1}$, Bo Xia ${ }^{1}$, Chenming $\mathrm{Hu}^{1}$, Li Yang ${ }^{1,2}$, Jialei Chen ${ }^{1}$, Longhuan $\mathrm{Du}^{3}$, Xiaosong Jiang ${ }^{1,2^{*}}$ \\ ${ }^{1}$ Sichuan Animal Science Academy, 610066, Chengdu, Sichuan, China \\ ${ }^{2}$ Animal Breeding and Genetics Key Laboratory of Sichuan Province, 610066, Chengdu, Sichuan, China \\ ${ }^{3}$ Sichuan University, 610000, Chengdu, Sichuan, China
}

\begin{abstract}
The introduction of forage grass in poultry industry had double benefit on animals and the environment. In this study, three kinds of pastures were planted artificially to evaluate their roles through the determination of grass, chicken and soil-related indexes. The results showed that raising chickens under the forest could destroy vegetation and affect the chemical properties of soil negatively. Changes of soil caused by chickens can be alleviated through planting grass. Forage provided a certain food source for chickens without affecting the growth, slaughter properties, meat quality, immune indexes and other performances. Suitable pasture should have good regeneration ability, trampling resistance, good palatability, and do not cause harm to the health of chickens.
\end{abstract}

\section{Introduction}

In recent years, free-range chicken production had a considerable scale in rural areas of China with the rising consumer demand for its products. It was a kind of broiler production which made full use of land and natural resources under the forest, or at the woodland, so as to cut down land, construction and feed cost. Chicken's manure was degraded naturally to provide nutrients for vegetation growth, which to some extent decreased manure disposal and fertilizer input. Freerange chicken foraged for pests, weeds and leftover crops while fertilizing and aerating the soil, which not only produced healthier eggs and meat than that locked up in a coop, but also created a symbiotic relationship between the animals and the land. Free-ranging was reported to improve the performance of chickens such as carcass yield and meat composition and it also took into account animal welfare ${ }^{1143}$.

However, overstocking of free-range chicken is bound to bring a variety of negative effects, such as vegetation destruction, soil compaction and even acidification. Grazing under different densities caused increase in different degrees in soil nutrients and acidification, and in high density flocks, no dominant plant existed, only the rhizomes of some grasses remained underground ${ }^{4}$. Fecal nitrogen and phosphorus input caused the accumulation of mineral nitrogen and phosphorus in soil. Phosohorus can loss from the soil via runoff or leaching, where environmental contamination occurred when land application of poultry wastes was in excess of crop utilization potential, or was done under poor management conditions. Meanwhile, it was possible to increase risk of exposure to environmental contaminants, parasites and potential pathogens through soil and plants intakes during exploration ${ }^{56}$. Besides, the possible negative effects of plant overintake on chicken performance are also worth considering.

Nowadays, the application of pasture grasses in the production of free-range poultry is increasingly becoming the focus of attention. Pasture grass can first of all act as a necessary supplement of food sources to reduce expensive feed inputs, especially for slow type chickens with long raising period. More importantly, it can effectively mitigate the damage to the ecological environment caused by the production of free-range chicken, for example, to maintain sufficient aboveground biomass and to alleviate soil nutrients enrichment and acidification. Although whether grazing is to the benefit of chickens' performance is still controversial, the promotion effect of some herbage on the growth performance of chickens has been proved. Legumebased pasture intake promoted bird performance while contributing to the production of broiler meat with preferred sensory attributes. Chicory was considered to be an ideal fiber-rich complementary and/or alternative livestock feed supplement for its high palatability and anti-parasitic activity in livestock ${ }^{6}$. Forage Rape (Brassica napus L.) is with good effect of feeding livestock and becomes an alternative to traditional feeds for dairy cows as it requires $40 \%$ less water than these latter in dairy semi-arid areas of Mexico ${ }^{8}$.

In this study, we compared three pasture grasses, Redroot Pigweed, Chicory and Forage Rape in terms of pastures growth performance, chicken growth performance and slaughter traits, as well as soil chemical

*Corresponding author: cwyang@foxmail.com; xsjiang2017@163.com 
properties, to evaluate the effect of pastures on environment and chickens, and furthermore to provide a reference for continuous screening of suitable pasture grasses and management of rotational grazing for freerange chicken production.

\section{Materials and methods}

\subsection{Experimental site and time}

The experiment was carried out in Daoming Town, Chengdu City of China $\left(103^{\circ} 07^{\prime} \mathrm{E} \sim 103^{\circ} 49^{\prime} \mathrm{E}, 30^{\circ} 30^{\prime}\right.$ $\mathrm{N} \sim 30^{\circ} 53^{\prime} \mathrm{N}$ ), with a subtropical monsoon climate, an annual rainfall of about 1,000 millimeters, and an annual average temperature of $16^{\circ} \mathrm{C}$. In July 2018, the experimental site was leveled and divided into four independent plots fenced with barbed wire, named Group A, Group B, Group C and Group D, which four were further divided into three pieces equally, with an area of 0.4 ha. Each group was artificially weeded for subsequent planting and stocking.

\subsection{Pasture planting}

Redroot Pigweed, Chicory and Forage Rape were provided by Sichuan Animal Science Academy, and was sown in Group A, Group B, Group C at July 2018, respectively. The grouping information, seeding rate and minimal plant height are shown in Table 1. Group D was treated as control.

Table 1. Seeding rate and required plant height of different forage varieties

\begin{tabular}{|l|l|l|}
\hline Forage / Group & Seeding rate $(\mathrm{kg} / \mathrm{ha})$ & Plant height $(\mathrm{cm})$ \\
\hline Redroot Pigweed & 7.50 & 40 \\
\hline Chicory & 12.00 & 35 \\
\hline Forage Rape & 15.00 & 40 \\
\hline Control & - & - \\
\hline
\end{tabular}

\subsection{Animals}

A total of 600 broilers (Dahen 699 broiler) bred by Sichuan Animal Science Academy were reared in coops during 0 to 6 weeks of age, and grazed from 7 to 18 weeks of age with 120 chickens per group. Each group was further repeated three times, with 40 chickens in each replicate. The feeding capacity and feed formulation at different growth stages are shown in Table 2. The procedures of feeding management and immunization were based on Guidance Manual for the Production of Free-range Chicken in Sichuan Province. The experiment was conducted in accordance with the Chinese guidelines for animal welfare and approved by the animal welfare committee of Sichuan Animal Science Academy.

Table 2. Feeding capacity and feed formulation at different growth stages

\begin{tabular}{|l|l|l|}
\hline $\begin{array}{l}\text { Growth stage } \\
\text { (weeks) }\end{array}$ & $\begin{array}{l}\text { Feed capacity } \\
\text { (g/d per chichen) }\end{array}$ & Feed formulation \\
\hline
\end{tabular}

\begin{tabular}{|l|l|l|}
\hline $0-6$ & ad libitum & $100 \%$ formula feed \\
\hline $7-8$ & $60-65$ & $100 \%$ formula feed \\
\hline $9-12$ & $68-80$ & $\begin{array}{l}80 \% \text { formula feed and } \\
20 \% \text { corn }\end{array}$ \\
\hline $13-18$ & $85-100$ & $\begin{array}{l}70 \% \text { formula feed and } \\
30 \% \text { corn }\end{array}$ \\
\hline
\end{tabular}

\subsection{Measurement of pasture growth performance}

The growth performance of pasture grass in each replicate was measured by five-point method at the end of each week during stocking period, with a sample plot size about $1 \mathrm{~m} \times 1 \mathrm{~m}$. The indexes included plant height, density and coverage, where the coverage was determined by line transect method with a transect length of $1.14 \mathrm{~m}$, and was calculated as follows:

$$
C=\frac{\sum B}{L} * 100
$$

, where $\mathrm{C}$ was coverage, $\mathrm{B}$ was total length of all species along transect, and $\mathrm{L}$ was transect length.

\subsection{Determination of indicators related chicken}

Production performance: The number of chickens, feed consumption and body weight of each chicken were recorded by replicates at the end of each week, and the culling and mortality rate (CMR), feed conversion ratio (FCR) and average body weight (BW) were calculated. Slaughter traits: At the end of 18 weeks, 20 chickens in each replicate were randomly selected for slaughter according to Performance Terms and Measurement for Poultry (NY/T 823). The indexes included live weight (LW), dressed weight (DW), eviscerated weight (EW), half-eviscerated weight (HEW), breast muscle weight (BMW), and leg muscle weight (LMW). The dressed percentage (DP), percentage of half-eviscerated yield (HEYP), percentage of eviscerated yield (EYP), percentage of breast muscle (BMP), and percentage of leg muscle (LMP) were further calculated.

Meat flavor character: While slaughter, 10 chickens in each replicate were randomly selected to sample the left breast muscle for detection of meat flavor character including the content of inosinic acid (IMP) and intramuscular fat (IMF) which was detected by HighPerformance Liquid Chromatography (HPLC) and Soxhlet extraction, respectively.

Immune parameters: The bursa of Fabricius and spleen were stripped and weighed while slaughtered for calculation of immune organ index.

\subsection{Testing of soil chemical properties}

0-20 cm soil samples in each replicate were collected by five-point sampling method at 3 time points, before seeding, at the beginning of stocking and at the end of stocking. The size of each sample plot was $10 \mathrm{~cm} \times 10$ $\mathrm{cm}$. The $\mathrm{pH}$ value was measured by Acidity Meter. The content of organic matter $(\mathrm{OM})$, total nitrogen $(\mathrm{TN})$, alkali-hydrolyzed nitrogen (AHN), available phosphorus (AP), and available potassium (AK) was tested by 
Potassium Bichromate Titrimetric Method, Ultraviolet and Visible Spectrophotometry, Alkaline Hydrolysis Diffusion Method, Sodium Molybdate Extraction and Molybdenum Antimony Colorimetric Method, and Ammonium Acetate Extraction-flame Photometry, respectively.

\subsection{Statistical analysis}

The results of indicators of pastures, chickens and soil were analyzed by SAS and illustrated in the form of 'mean \pm standard deviation'. Means were compared and significance was declared based on least significant difference at probability by $\mathrm{T}$-test, $\mathrm{P} \leq 0.05$.

\section{Results}

\subsection{Change of pasture growth performance}

The growth performance data of different pasture are shown in Table 3, 4 and 5. The plant height and density of Forage Rape and Chicory decreased rapidly, lower than $5 \mathrm{~cm}$ at 14 and 12 weeks of age, respectively, and remained only underground stems or root tubers at 16 and 14 weeks of age, respectively. However, those of Redroot Pigweed changed little with the increase of stocking time. The coverage of all the three forage groups decreased sharply. With $20 \%$ coverage as a reference, Redroot Pigweed sustained to the end of the experiment, while that of Forage Rape and Chicory only lasted to 13 and 11 weeks, respectively.

Table 3. The plant height of pastures $(\mathrm{cm})$

\begin{tabular}{|c|c|c|c|}
\hline $\begin{array}{l}\text { Weeks } \\
\text { of age }\end{array}$ & Redroot Pigweed & Chicory & Forage Rape \\
\hline 6 & $83.45 \pm 8.37^{\mathrm{b}}$ & $35.28 \pm 4.63^{\mathrm{a}}$ & $54.68 \pm 22.14^{\mathrm{a}}$ \\
\hline 7 & $84.32 \pm 8.20^{\mathrm{ab}}$ & $32.30 \pm 3.63^{\mathrm{ab}}$ & $52.15 \pm 19.13^{\mathrm{a}}$ \\
\hline 8 & $86.18 \pm 10.66^{\mathrm{ab}}$ & $26.88 \pm 2.84^{\mathrm{b}}$ & $45.88 \pm 20.15^{\mathrm{b}}$ \\
\hline 9 & $89.04 \pm 10.42^{\mathrm{a}}$ & $17.46 \pm 3.01^{\mathrm{c}}$ & $32.66 \pm 20.55^{\mathrm{c}}$ \\
\hline 10 & $87.42 \pm 13.83^{\mathrm{ab}}$ & $10.44 \pm 3.20^{\mathrm{d}}$ & $26.69 \pm 15.94^{\mathrm{d}}$ \\
\hline 11 & $85.30 \pm 12.60^{\mathrm{ab}}$ & $6.20 \pm 3.37^{\mathrm{e}}$ & $17.28 \pm 13.46^{\mathrm{e}}$ \\
\hline \multicolumn{4}{|c|}{$\begin{array}{l}\text { The same lowercase letters in columns indicated that there was no } \\
\text { significant difference }(\mathrm{p}>0.05) \text {, and different lowercase letters indicatec } \\
\text { significant differences }(\mathrm{p}<0.05) \text {. } \\
\text { The same as Table } 4 \text { and Table } 5 \text {. }\end{array}$} \\
\hline
\end{tabular}

Table 4. The density of pastures(plant $\left./ \mathrm{m}^{2}\right)$

\begin{tabular}{|c|c|c|c|}
\hline $\begin{array}{c}\text { Weeks } \\
\text { of age }\end{array}$ & Redroot Pigweed & Chicory & Forage Rape \\
\hline 6 & $30.20 \pm 4.28^{\mathrm{a}}$ & $28.74 \pm 5.48^{\mathrm{a}}$ & $84.60 \pm 10.23^{\mathrm{a}}$ \\
\hline 7 & $28.40 \pm 6.56^{\mathrm{a}}$ & $26.40 \pm 4.67^{\mathrm{ab}}$ & $83.80 \pm 9.53^{\mathrm{a}}$ \\
\hline 8 & $28.20 \pm 1.33^{\mathrm{a}}$ & $25.20 \pm 6.18^{\mathrm{ab}}$ & $75.00 \pm 9.91^{\mathrm{b}}$ \\
\hline 9 & $25.60 \pm 3.61^{\mathrm{ab}}$ & $24.80 \pm 6.27^{\mathrm{b}}$ & $61.40 \pm 5.02^{\mathrm{c}}$ \\
\hline 10 & $22.20 \pm 2.32^{\mathrm{ab}}$ & $20.40 \pm 4.08^{\mathrm{c}}$ & $40.40 \pm 8.14^{\mathrm{d}}$ \\
\hline 11 & $20.00 \pm 1.67^{\mathrm{bcd}}$ & $17.20 \pm 2.99^{\mathrm{c}}$ & $18.60 \pm 4.10^{\mathrm{e}}$ \\
\hline
\end{tabular}

Table 5. The coverage of pastures(\%)

\begin{tabular}{|c|c|c|c|}
\hline $\begin{array}{c}\text { Weeks } \\
\text { of age }\end{array}$ & Redroot Pigweed & Chicory & Forage Rape \\
\hline 6 & $96.52 \pm 2.78^{\mathrm{a}}$ & $98.19 \pm 1.02^{\mathrm{a}}$ & $84.60 \pm 10.23^{\mathrm{a}}$ \\
\hline 7 & $95.60 \pm 4.09^{\mathrm{a}}$ & $97.10 \pm 1.56^{\mathrm{a}}$ & $83.80 \pm 9.53^{\mathrm{a}}$ \\
\hline 8 & $93.80 \pm 6.94^{\mathrm{a}}$ & $92.00 \pm 5.55^{\mathrm{a}}$ & $75.00 \pm 9.91^{\mathrm{b}}$ \\
\hline
\end{tabular}

\begin{tabular}{|c|c|c|c|}
\hline 9 & $82.70 \pm 4.26^{\mathrm{b}}$ & $79.90 \pm 7.59^{\mathrm{b}}$ & $61.40 \pm 5.02^{\mathrm{c}}$ \\
\hline 10 & $65.20 \pm 10.53^{\mathrm{c}}$ & $55.10 \pm 7.26^{\mathrm{c}}$ & $40.40 \pm 8.14^{\mathrm{d}}$ \\
\hline 11 & $48.30 \pm 8.69^{\mathrm{d}}$ & $37.00 \pm 9.49^{\mathrm{d}}$ & $18.60 \pm 4.10^{\mathrm{e}}$ \\
\hline
\end{tabular}

\subsection{Comparison of chicken growth performance}

The data of BW are shown in Table 6. The weight of chickens in different forage groups changed differently. Compared with Redroot Pigweed Group and Control Group, the BW of the chickens in Forage Rape Group and Chicory Group increased faster. However, at the end of stocking, the BW in Chicory Group was larger than that in Forage Rape Group with no significant difference, and was significantly higher than that in Redroot Pigweed Group and Control Group.

With the extension of stocking time, FCR increased gradually and followed a pattern among groups as fellow: Chicory Group (3.74) < Forage Rape Group (4.03) < Redroot Pigweed Group (4.22) < Control Group (4.44).

The slaughter traits data are shown in Table 7 . The inter-comparison of LMW, DW, DP and HEYP showed significant differences among some groups, eg. LMW in Chicory Group and DP in Redroot Pigweed Group vs Control Group, and HEYP in Redroot Pigweed Group and Forage Rape Group vs Chicory Group and Control Group. There was no significant difference in EYP, BMP and LMP among groups.

Table 6. Test results of body weight $(\mathrm{g})$

\begin{tabular}{|c|c|c|c|c|}
\hline $\begin{array}{l}\text { Weeks } \\
\text { of age }\end{array}$ & $\begin{array}{l}\text { Redroot } \\
\text { Pigweed }\end{array}$ & Chicory & $\begin{array}{l}\text { Forage } \\
\text { Rape } \\
\end{array}$ & Control \\
\hline 6 & $\begin{array}{l}1061.72 \\
\pm 93.22^{\mathrm{a}} \\
\end{array}$ & $\begin{array}{l}1064.97 \\
\pm 101.22^{\mathrm{a}} \\
\end{array}$ & $\begin{array}{l}1083.33 \\
\pm 98.12^{\mathrm{a}} \\
\end{array}$ & $\begin{array}{l}1074.38 \\
\pm 98.37^{\mathrm{a}} \\
\end{array}$ \\
\hline 7 & $\begin{array}{l}1139.06 \\
\pm 157.45^{\mathrm{a}} \\
\end{array}$ & $\begin{array}{l}1232.53 \\
\pm 112.33^{\mathrm{a}} \\
\end{array}$ & $\begin{array}{l}1220.82 \\
\pm 161.94^{\mathrm{a}} \\
\end{array}$ & $\begin{array}{l}1158.94 \\
\pm 134.58^{\mathrm{a}} \\
\end{array}$ \\
\hline 8 & $\begin{array}{l}1241.06 \\
\pm 142.45^{\mathrm{a}} \\
\end{array}$ & $\begin{array}{l}1439.25 \\
\pm 142.35^{\mathrm{b}} \\
\end{array}$ & $\begin{array}{l}1360.62 \\
\pm 141.64^{\text {ab }}\end{array}$ & $\begin{array}{l}1230.94 \\
\pm 113.45^{a} \\
\end{array}$ \\
\hline 9 & $\begin{array}{l}1376.01 \\
\pm 183.62^{\mathrm{a}} \\
\end{array}$ & $\begin{array}{l}1582.77 \\
\pm 127.65^{\mathrm{c}}\end{array}$ & $\begin{array}{l}1504.01 \\
\pm 177.29^{\mathrm{b}}\end{array}$ & $\begin{array}{l}1329.44 \\
\pm 155.66^{\mathrm{a}}\end{array}$ \\
\hline 10 & $\begin{array}{l}1439.52 \\
\pm 192.18^{\mathrm{a}} \\
\end{array}$ & $\begin{array}{l}1689.75 \\
\pm 153.81^{\mathrm{c}} \\
\end{array}$ & $\begin{array}{l}1618.35 \\
\pm 212.36^{\mathrm{b}} \\
\end{array}$ & $\begin{array}{l}1413.58 \\
\pm 188.64 \\
\end{array}$ \\
\hline 11 & $\begin{array}{l}1520.49 \\
\pm 213.13^{\mathrm{a}} \\
\end{array}$ & $\begin{array}{l}1776.09 \\
\pm 182.63^{\mathrm{b}} \\
\end{array}$ & $\begin{array}{l}1700.58 \\
\pm 213.68^{\mathrm{b}} \\
\end{array}$ & $\begin{array}{l}1439.58 \\
\pm 245.87^{a} \\
\end{array}$ \\
\hline 12 & $\begin{array}{l}1603.27 \\
\pm 243.29^{\mathrm{a}} \\
\end{array}$ & $\begin{array}{l}1888.57 \\
\pm 198.47^{\mathrm{c}} \\
\end{array}$ & $\begin{array}{l}1787.07 \\
\pm 224.23^{\mathrm{b}} \\
\end{array}$ & $\begin{array}{l}1563.69 \\
\pm 221.29^{a} \\
\end{array}$ \\
\hline 13 & $\begin{array}{l}1761.65 \\
\pm 248.37^{\mathrm{b}} \\
\end{array}$ & $\begin{array}{l}1951.8 \\
\pm 212.55^{\mathrm{d}} \\
\end{array}$ & $\begin{array}{l}1861.66 \\
\pm 278.63^{\mathrm{c}} \\
\end{array}$ & $\begin{array}{l}1667.00 \\
\pm 236.38^{\mathrm{a}} \\
\end{array}$ \\
\hline 14 & $\begin{array}{l}1841.43 \\
\pm 278.42^{\mathrm{a}} \\
\end{array}$ & $\begin{array}{l}2073.58 \\
\pm 223.78^{\mathrm{c}}\end{array}$ & $\begin{array}{l}1957.14 \\
\pm 285.96^{b}\end{array}$ & $\begin{array}{l}1774.46 \\
\pm 294.63^{a} \\
\end{array}$ \\
\hline 15 & $\begin{array}{l}1954.82 \\
\pm 285.49^{\mathrm{a}} \\
\end{array}$ & $\begin{array}{l}2144.84 \\
\pm 229.34^{\mathrm{c}} \\
\end{array}$ & $\begin{array}{l}2063.72 \\
\pm 294.39^{\mathrm{b}}\end{array}$ & $\begin{array}{l}1900.92 \\
\pm 246.75^{a} \\
\end{array}$ \\
\hline 16 & $\begin{array}{l}2052.38 \\
\pm 292.64^{\mathrm{a}} \\
\end{array}$ & $\begin{array}{l}2259.33 \\
\pm 334.61^{\mathrm{b}} \\
\end{array}$ & $\begin{array}{l}2192.5 \\
\pm 317.44^{\mathrm{b}}\end{array}$ & $\begin{array}{l}2030.11 \\
\pm 278.55^{\mathrm{a}} \\
\end{array}$ \\
\hline 17 & $\begin{array}{l}2208.82 \\
\pm 313.78^{\mathrm{ab}}\end{array}$ & $\begin{array}{l}2373.76 \\
\pm 421.37^{\mathrm{c}} \\
\end{array}$ & $\begin{array}{l}2295.87 \\
\pm 368.69^{\mathrm{bc}}\end{array}$ & $\begin{array}{l}2198.32 \\
\pm 366.74^{\mathrm{a}}\end{array}$ \\
\hline 18 & $\begin{array}{l}2358.66 \\
\pm 295.19^{\text {a }} \\
\end{array}$ & $\begin{array}{l}2494.24 \\
\pm 454.94^{\mathrm{b}} \\
\end{array}$ & $\begin{array}{l}2430.36 \\
\pm 373.73^{\text {ab }} \\
\end{array}$ & $\begin{array}{l}2320.23 \\
\pm 234.12^{\mathrm{a}} \\
\end{array}$ \\
\hline \multicolumn{5}{|c|}{$\begin{array}{l}\text { The same index is compared between different groups in the same } \\
\text { row, the same lowercase letters indicate that the difference is not } \\
\text { significant }(p>0.05) \text {, different lowercase letters indicate significant } \\
\text { differences }(p<0.05) \text {. } \\
\text { The same as Table } 7,8 \text { and } 9 \text {. }\end{array}$} \\
\hline
\end{tabular}

Table 7. Test results of slaughter performances

\begin{tabular}{|l|l|l|l|l|}
\hline Traits & $\begin{array}{l}\text { Redroot } \\
\text { Pigweed }\end{array}$ & Chicory & $\begin{array}{l}\text { Forage } \\
\text { Rape }\end{array}$ & Control \\
\hline LW & 2358.66 & 2494.24 & 2430.36 & 2320.23 \\
$\pm 295.19^{\mathrm{a}}$ & $\pm 454.94^{\mathrm{b}}$ & $\pm 373.73^{\mathrm{ab}}$ & $\pm 234.12^{\mathrm{a}}$ \\
\hline
\end{tabular}




\begin{tabular}{|l|l|l|l|l|}
\hline \multirow{2}{*}{ DW } & $\begin{array}{l}2163.12 \\
\pm 234.25^{\mathrm{a}}\end{array}$ & $\begin{array}{l}2252.99 \\
\pm 323.25^{\mathrm{b}}\end{array}$ & $\begin{array}{l}2211.05 \\
\pm 218.65^{\mathrm{ab}}\end{array}$ & $\begin{array}{l}2075.41 \\
\pm 221.55^{\mathrm{c}}\end{array}$ \\
\hline \multirow{2}{*}{ DP } & 91.71 & 90.33 & 90.98 & 89.45 \\
& $\pm 10.25^{\mathrm{a}}$ & $\pm 10.10^{\mathrm{abc}}$ & $\pm 9.28^{\mathrm{ab}}$ & $\pm 8.12^{\mathrm{c}}$ \\
\hline \multirow{2}{*}{ HEW } & 84.49 & 82.57 & 83.92 & 82.88 \\
& $\pm 9.73^{\mathrm{a}}$ & $\pm 9.33^{\mathrm{b}}$ & $\pm 9.74^{\mathrm{a}}$ & $\pm 6.37^{\mathrm{b}}$ \\
\hline \multirow{2}{*}{ EWP } & 71.28 & 69.44 & 70.13 & 69.55 \\
& $\pm 8.66^{\mathrm{a}}$ & $\pm 7.14^{\mathrm{a}}$ & $\pm 6.35^{\mathrm{a}}$ & $\pm 7.02^{\mathrm{a}}$ \\
\hline \multirow{2}{*}{ BMWP } & 14.32 & 14.49 & 13.96 & 14.83 \\
& $\pm 1.57^{\mathrm{a}}$ & $\pm 1.07^{\mathrm{a}}$ & $\pm 1.02^{\mathrm{a}}$ & $\pm 1.69^{\mathrm{a}}$ \\
\hline \multirow{2}{*}{ LMWP } & 23.45 & 23.98 & 23.49 & 23.78 \\
& $\pm 3.24^{\mathrm{a}}$ & $\pm 2.05^{\mathrm{a}}$ & $\pm 1.64^{\mathrm{a}}$ & $\pm 2.10^{\mathrm{a}}$ \\
\hline LW, live weight(kg); DW, dressed weight(kg); DP, dressing \\
percentage(\%); HEWP, percentage of half-eviscerated weight $(\%) ;$ \\
EWP, percentage of eviscerated weight(\%); BMWP, percentage of \\
breast muscle weight(\%); LMWP, percentage of leg muscle \\
weight(\%).
\end{tabular}

\subsection{Difference in meat quality traits and immune index}

The results of IMP content and IMF content are shown in Table 8, and the index of immune organs are compared in Table 9. The meat quality traits and bursa of Fabricius index were with no significant difference among groups, while the spleen index in Chicory Group and Control Group was significantly higher than that in Redroot Pigweed Group and Forage Rape Group.

Table 8. Test results of meat quality traits

\begin{tabular}{|l|l|l|l|l|}
\hline Traits & $\begin{array}{l}\text { Redroot } \\
\text { Pigweed }\end{array}$ & Chicory & $\begin{array}{l}\text { Forage } \\
\text { Rape }\end{array}$ & Control \\
\hline IMP & 1.846 & 1.823 & 1.878 & 1.850 \\
& $\pm 0.204^{\mathrm{a}}$ & $\pm 0.185^{\mathrm{a}}$ & $\pm 0.194^{\mathrm{a}}$ & $\pm 0.201^{\mathrm{a}}$ \\
\hline IMF & 1.042 & 1.038 & 1.015 & 1.027 \\
& $\pm 0.200^{\mathrm{a}}$ & $\pm 0.167^{\mathrm{a}}$ & $\pm 0.114^{\mathrm{a}}$ & $\pm 0.135^{\mathrm{a}}$ \\
\hline \multicolumn{4}{|l}{ IMP, Inosinic acid content(mg/g); IMF, Intramuscular fat content $(\%)}$. \\
\hline
\end{tabular}

Table 9. Test results of immune indexes

\begin{tabular}{|c|c|c|c|c|}
\hline Traits & $\begin{array}{l}\text { Redroot } \\
\text { Pigweed }\end{array}$ & Chicory & $\begin{array}{l}\text { Forage } \\
\text { Rape }\end{array}$ & Control \\
\hline $\begin{array}{l}\text { Spleen } \\
\text { index }\end{array}$ & $\begin{array}{l}0.14 \\
\pm 0.010^{\mathrm{a}}\end{array}$ & $\begin{array}{l}0.16 \\
\pm 0.003^{b}\end{array}$ & $\begin{array}{l}0.13 \\
\pm 0.007^{\mathrm{a}}\end{array}$ & $\begin{array}{l}0.17 \\
\pm 0.010^{b}\end{array}$ \\
\hline $\begin{array}{l}\text { BF } \\
\text { index }\end{array}$ & $\begin{array}{l}0.07 \\
\pm 0.002^{\mathrm{a}}\end{array}$ & $\begin{array}{l}0.06 \\
\pm 0.002^{\mathrm{a}}\end{array}$ & $\begin{array}{l}0.06 \\
\pm 0.003^{\mathrm{a}}\end{array}$ & $\begin{array}{l}0.07 \\
\pm 0.005^{\mathrm{a}}\end{array}$ \\
\hline CMR & $\begin{array}{l}10.23 \\
\pm 1.02^{\mathrm{a}}\end{array}$ & $\begin{array}{l}9.74 \\
\pm 0.95^{\mathrm{a}}\end{array}$ & $\begin{array}{l}12.23 \\
\pm 0.89^{\mathrm{a}}\end{array}$ & $\begin{array}{l}10.15 \\
\pm 0.54^{\mathrm{a}}\end{array}$ \\
\hline
\end{tabular}

\subsection{Variation of soil chemical properties}

The detection results of soil chemical properties are shown in Table 10.

Table 10. Test results of soil physical and chemical properties

\begin{tabular}{|l|l|l|l|l|l|l|}
\hline \multicolumn{7}{|l|}{ Before seeding } \\
\hline Groups & $\mathrm{pH}$ & $\mathrm{OM}$ & $\mathrm{TN}$ & $\mathrm{AHN}$ & $\mathrm{AP}$ & $\mathrm{AK}$ \\
\hline Redroot & $7.9 \pm$ & $24.4 \pm 2$. & $0.8 \pm$ & $95.8^{\mathrm{Aa}}$ & $22.6 \pm$ & $94.2 \pm$ \\
Pigweed & $0.5^{\mathrm{Aa}}$ & $3^{\mathrm{Aa}}$ & $0.1^{\mathrm{Aa}}$ & $3.7^{\mathrm{Aa}}$ & $1.4^{\mathrm{Aa}}$ & $2.8^{\mathrm{Aa}}$ \\
\hline \multirow{2}{*}{ Chicory } & $7.8 \pm$ & $24.4 \pm 2$. & $0.8 \pm$ & $96.8 \pm$ & $24.1 \pm$ & $95.4 \pm$ \\
& $0.3^{\mathrm{Aa}}$ & $1^{\mathrm{Aa}}$ & $0.1^{\mathrm{Aa}}$ & $3.9^{\mathrm{Aa}}$ & $2.7^{\mathrm{Aa}}$ & $5.8^{\mathrm{Aa}}$ \\
\hline Forage & $7.9 \pm$ & $22.9 \pm 1$. & $0.2 \pm$ & $100.0 \pm$ & $23.8 \pm$ & $91.8 \pm$ \\
Rape & $0.7^{\mathrm{Aa}}$ & $5^{\mathrm{Aa}}$ & $0.0^{\mathrm{Aa}}$ & $2.4^{\mathrm{Aa}}$ & $2.6^{\mathrm{Aa}}$ & $4.7^{\mathrm{Aa}}$ \\
\hline \multirow{2}{*}{ Control } & $7.2 \pm$ & $24.7 \pm 1$. & $0.7 \pm$ & $97.0 \pm$ & $23.8 \pm$ & $98.4 \pm$ \\
& $0.8^{\mathrm{Aa}}$ & $3^{\mathrm{Aa}}$ & $0.8^{\mathrm{Aa}}$ & $2.4^{\mathrm{Aa}}$ & $1.5^{\mathrm{Aa}}$ & $3.9^{\mathrm{Aa}}$ \\
\hline At the beginning of stocking \\
\hline Redroot & $7.9 \pm$ & $21.4 \pm 1$. & $0.8 \pm$ & $77.2 \pm$ & $25.6 \pm$ & $91.6 \pm$ \\
Pigweed & $0.5^{\mathrm{Aa}}$ & $5^{\mathrm{Bb}}$ & $0.1^{\mathrm{Aa}}$ & $4.8^{\mathrm{Bb}}$ & $3.6^{\mathrm{Aa}}$ & $5.8^{\mathrm{Ba}}$ \\
\hline
\end{tabular}

\begin{tabular}{|c|c|c|c|c|c|c|}
\hline Chicory & $\begin{array}{l}7.5^{ \pm} \\
0.3^{\mathrm{Aa}} \\
\end{array}$ & $\begin{array}{l}23.4 \pm 1 . \\
3^{\mathrm{Aa}}\end{array}$ & $\begin{array}{l}0.8^{ \pm} \\
0.0^{\mathrm{Ba}} \\
\end{array}$ & $\begin{array}{l}76.6 \pm \\
5.6^{\mathrm{Bb}} \\
\end{array}$ & $\begin{array}{l}20.1 \pm \\
2.6^{\mathrm{Aa}}\end{array}$ & $\begin{array}{l}99.6 \pm \\
4.8^{\mathrm{Aa}}\end{array}$ \\
\hline $\begin{array}{l}\text { Forage } \\
\text { Rape }\end{array}$ & $\begin{array}{l}7.9 \pm \\
0.4^{\mathrm{Aa}}\end{array}$ & $\begin{array}{l}23.9 \pm 1 \\
2^{\mathrm{Aa}}\end{array}$ & $\begin{array}{l}0.8^{ \pm} \\
0.1^{\mathrm{Aa}}\end{array}$ & $\begin{array}{l}95.6 \pm \\
8.7^{\text {Aa }}\end{array}$ & $\begin{array}{l}24.0 \pm \\
1.5^{\mathrm{Aa}}\end{array}$ & $\begin{array}{l}97.2 \pm \\
7.7^{\mathrm{Aa}}\end{array}$ \\
\hline $\begin{array}{l}\text { Redroot } \\
\text { Pigweed }\end{array}$ & $\begin{array}{l}7.6 \pm \\
0.6^{\mathrm{Aa}} \\
\end{array}$ & $\begin{array}{l}24.1 \pm 1 . \\
2^{\mathrm{Aa}}\end{array}$ & $\begin{array}{l}0.9 \pm \\
0.0^{\mathrm{Aa}} \\
\end{array}$ & $\begin{array}{l}101.4 \pm \\
4.0^{\mathrm{Aa}}\end{array}$ & $\begin{array}{l}23.2 \pm \\
1.5^{\mathrm{Aa}} \\
\end{array}$ & $\begin{array}{l}101.2 \pm \\
6.9^{\mathrm{Aa}}\end{array}$ \\
\hline \multicolumn{7}{|c|}{ At the end of stocking } \\
\hline $\begin{array}{l}\text { Redroot } \\
\text { Pigweed }\end{array}$ & $\begin{array}{l}7.7 \pm \\
0.9^{\mathrm{Ba}} \\
\end{array}$ & $\begin{array}{l}23.4 \pm 3 . \\
2^{\mathrm{Ba}}\end{array}$ & $\begin{array}{l}1.2 \pm \\
0.2^{\mathrm{Bb}} \\
\end{array}$ & $\begin{array}{l}123.2 \pm \\
9.9^{\mathrm{Bc}} \\
\end{array}$ & $\begin{array}{l}47.4 \pm \\
3.8^{\mathrm{Bb}} \\
\end{array}$ & $\begin{array}{l}108.2 \pm \\
13.9^{\mathrm{Bb}} \\
\end{array}$ \\
\hline Chicory & $\begin{array}{l}7.0 \pm \\
0.9^{\mathrm{cb}} \\
\end{array}$ & $\begin{array}{l}30.2 \\
\pm 2.2^{\mathrm{Cb}}\end{array}$ & $\begin{array}{l}1.0 \pm \\
0.2^{\mathrm{Cb}} \\
\end{array}$ & $\begin{array}{l}125.4^{ \pm} \\
10.4^{\mathrm{Cc}}\end{array}$ & $\begin{array}{l}55.4 \pm \\
4.1^{\mathrm{cb}}\end{array}$ & $\begin{array}{l}153.0 \pm \\
11.4^{\mathrm{Cb}} \\
\end{array}$ \\
\hline $\begin{array}{l}\text { Forage } \\
\text { Rape }\end{array}$ & $\begin{array}{l}7.3^{ \pm} \\
0.9^{\mathrm{cb}} \\
\end{array}$ & $\begin{array}{l}25.9 \pm 2 . \\
7^{\mathrm{Db}}\end{array}$ & $\begin{array}{l}1.5^{ \pm} \\
0.1^{\mathrm{cb}} \\
\end{array}$ & $\begin{array}{l}152.2 \pm \\
11.6^{\mathrm{Db}}\end{array}$ & $\begin{array}{l}65.4 \pm \\
4.2^{\mathrm{Db}}\end{array}$ & $\begin{array}{l}149.4 \pm \\
9.5^{\mathrm{Cb}} \\
\end{array}$ \\
\hline $\begin{array}{l}\text { Redroot } \\
\text { Pigweed }\end{array}$ & $\begin{array}{l}6.6 \pm \\
1.0^{\mathrm{Ab}} \\
\end{array}$ & $\begin{array}{l}35.2 \pm 4 . \\
9^{\mathrm{Ab}}\end{array}$ & $\begin{array}{l}1.8^{ \pm} \\
0.3^{\mathrm{Ab}} \\
\end{array}$ & $\begin{array}{l}226.8 \pm \\
36.9^{\mathrm{Ab}} \\
\end{array}$ & $\begin{array}{l}72.8 \pm \\
5.7^{\mathrm{Ab}} \\
\end{array}$ & $\begin{array}{l}173.4 \pm \\
16.5^{\mathrm{Ab}} \\
\end{array}$ \\
\hline \multicolumn{7}{|c|}{$\begin{array}{l}\mathrm{OM} \text {, organic matter }(\mathrm{g} / \mathrm{kg}) ; \mathrm{TN} \text {, total nitrogen }(\mathrm{g} / \mathrm{kg}) ; \mathrm{AHN} \text {, alkali hydrolyzable } \\
\text { nitrogen }(\mathrm{mg} / \mathrm{kg}) ; \mathrm{AP} \text {, available phosphorus }(\mathrm{mg} / \mathrm{kg}) ; \mathrm{AK} \text {, available } \\
\text { potassium }(\mathrm{mg} / \mathrm{kg}) \text {. } \\
\text { Uppercase letters indicate comparison of the same index at the same time between } \\
\text { different groups. The same uppercase letters indicate that the difference is not } \\
\text { significant, different uppercase letters indicate significant differences }(\mathrm{p}<0.05) \text {. } \\
\text { Lowercase letters indicate comparison of the same index at different time between } \\
\text { different groups. The same lowercase letters indicate that the difference is not } \\
\text { significant, different lowercase letters indicate significant differences }(\mathrm{p}<0.05) \text {. }\end{array}$} \\
\hline
\end{tabular}

Comparison of the soil chemical properties among groups before seeding showed a homogeneous soil condition. According to the results at the beginning of stocking, the growth of pastures brought no change in $\mathrm{pH}$ and content of AP, but changed the content of OM, $\mathrm{AK}, \mathrm{TN}$ and AHN in some groups significantly. At the end of stocking, the content of OM, TN, AHN, AP and $\mathrm{AK}$ in pasture groups were significantly lower than those in Control Group, while $\mathrm{pH}$ value displayed a contrary result.

As the experiment went on, the variation trend of soil chemical properties in different herbage groups were distinct. Compared with the indexes of each group before seeding and at the beginning of stocking, no significant changes was observed except a significant decrease of the OM and ANH consistent in Redroot Pigweed Group, and the AHN content in Chicory Group. Meanwhile, comparison between the data at the beginning of stocking and at the end of stocking by groups illustrated a significant rise in the content of OM, $\mathrm{TN}, \mathrm{AHN}, \mathrm{AP}$ and $\mathrm{AK}$, and an obvious decrease in $\mathrm{pH}$ value, especially in Control Group, Chicory Group and Forage Rape Group.

\section{Discussion and conclusions}

The free-range chicken production will be around for a long time. It is in line with the sustainable development demand of a large number of farmers, agriculture and rural area in China. However, if this kind of production is not properly managed, it will inevitably cause a range of issues, for instance, environmental pollution and food safety, and reduction of producers' profits. Introduction of forage grass is an effective alternative to alleviate the disadvantages above. Good forage grass can take into account both the interests of chickens and environment to avoid direct conflict between them. However, considered the digestive properties of birds, the growth properties of plants, it is necessary to screen suitable forages based on accurate grasp of the interaction among pasture, chicken and environment.

The evaluation of suitable herbage for free-range chicken production should take into account the nutritional value and palatability of herbage, the 
influence on production performance of chickens and the prevention of environmental damage. The content of crude protein $(\mathrm{CP})$ and crude fiber(CF) were the indicators of priority we focused on, because 1) forages can provide CP as a source of protein in diet, and 2) the utilization of forage is limited by $\mathrm{CF}$ due to the limit digestibility coefficient by chickens. However, a herbage's nutritional value varied with species, organs, growing stages and management plan, etc., so we made a preliminary assessment by literature reviews instead of measuring in this experiment. The $\mathrm{CP}$ content and $\mathrm{CF}$ content of Chicory, Redroot Pigweed and Forage Rape according to the review was about $14.70 \%, 27.07 \%$, $17.26 \%$, and $16.78 \%, 9.32 \%$, and $27.80 \%$ on dry weight basis, respectively ${ }^{91011}$. It should be noted that the data had no comparative significance and were for reference only because of the different material, different testing method, and different source of test-data. Redroot Pigweed seems to be the best for its highest CP content and lowest CF content. However, according to previous researches, it may cause fatal nephrotoxicity and other symptoms, such as bloat, which presumably because of its high oxalate content. Thus, it may be harmful and even deadly when fed to some animals in large amounts over several days, eg. in lamb, swine and cattle Nevertheless, it was reported inability to cause renal toxicosis in rabbits, and we did not retrieve report about toxicity of amaranth to chickens. In fact, oxalates are ubiquitous in all kinds of plants, and Chicory and Forage Rape planted in this experiment were no exception. In order to reduce the possibility of adverse effects from excessive plant consumption, we supplemented the chickens with formula feed and corn to reduce the plant portion of diet.

Palatability refers to chicken's enthusiasm for eating plants. It is influenced by plant secondary metabolites (PSM), such as tannins and phenols, which can present bitter and astringent tastes. Meanwhile, sesquiterpene lactones (SL) present in Chicory leaves (about $0.2 \%$ in Dry Matter) is also responsible for its bitter taste. Chicory has previously been referred to "tanniniferous forage", although only low levels of condensed tannins (less than $0.8 \%$ in Dry Matter) are commonly detected in leaves. Tannin levels from $0.5 \%-2.0 \%$ was reported to suppress growth and egg production in poultry, and between 3\% - 7\% inclusion levels in diet may be lethal. Therefore, we also recognized that the use of Chicory in chicken production may be limited due to the bad palatability and negative impact to chickens. Interestingly, according to our observation, flocks in Chicory group took slightly longer to eat up their feed than that in other groups. It probably meant that chickens preferred Chicory rather than other feed we provided, and bitterness of Chicory did not affect the plant intake. Thus, Redroot Pigweed, Chicory and Forage Rape were all proved to have good palatability.

As mentioned above, the impact of pastures to chickens' healthy is a top-priority factor and we evaluated it based on indicators including growth performance, slaughter performance, meat quality and so on. In this study, herbage seem to have good effects (eg. the BW in Chicory Group), or no improvement on some of indexes above instead of negative effects. However, we neither further explored the causality or mechanism, for example, the relationship between Chicory intake and weight gain, nor detected the changes at the molecular level or organopathological changes in chickens that might caused by herbage. In fact, the health-promoting activities of Chicory, such as anti-inflammatory, antiviral, antibacterial, antifungal, immune-stimulating and anthelmintic, had been reviewed, and the improvement to chickens' performance was also been reported ${ }^{12]}$. Combined with the results of herbage growth performance, it can also be inferred that the live weight of chickens can be significantly improved under the premise of sufficient yield in Chicory group and Forage Rape group. The stocking period of free-range production was relatively long than that in cages, which led to more feed consumption, and therefore the positive effect of pastures on FCR was of practical significance. We supplemented the flocks with full-price feed and herbage, and the FCR comparison illustrated that different pastures had different positive effect on FCR, which reduced the feed consumption and furthermore decreased production cost.

Forage grass intake increased the chicken's satiety and met their feeding habit, so as to following animal welfare demand. However, the trampling of poultry on woodland led to tree roots exposure, vegetation destruction, soil compaction, soil bulk density increase and porosity reduction, thus causing soil and water loss ${ }^{4}$. We considered and evaluated the trampling resistance through forage growth performance. Redroot Pigweed showed the best trampling resistance, while Chicory and Forage Rape was influenced seriously and showed bad trampling. However, based on the regeneration of herbage, the problem of vegetation damage can be solved by rotation grazing in practical production.

Early studies had determined the ability of Redroot Pigweed to extract $137 \mathrm{Cs}$ and $90 \mathrm{Sr}$ from contaminated soil, with a concentration ratios about 2.58 and 6.5, respectively ${ }^{13}$. Chicory may have high $\mathrm{N}$ uptake efficiency to improve the management of intensive dairy farms with a large $\mathrm{N}$ surplus and add significant improvements of the quality of the forage. Forage Rape is a valuable cover crop to prevent soil erosion and $\mathrm{N}$ leaching, improve soil structure as green manure during end of summer, autumn and winter ${ }^{14]}$. Our preliminary study and the determination of soil chemical properties in this experiment also confirmed that the production of chickens had a bad effect on soil. However, the application of herbage can alleviate these effects, and Redroot Pigweed had obvious advantages in this aspect.

\section{Acknowledgments}

We are grateful to the editors and reviewers for the careful reviews and the honest advice. Particular thanks go to all of the people for their help with sample collection and laboratory analysis. This work was supported by Chinese National Agriculture Research System (CARS-41-G04), Key Technology Support Program of Sichuan Province (2019YFN0009, 
2021YFYZ0031, 21ZDYF2278), and Sichuan Provincial Finance Operation Special Project (SASA2020CZYX002, SASA2020CZYX007).

\section{Reference}

1. Koçer B, Bozkurt M, Ege G, et al. Effects of a meal feeding regimen and the availability of fresh alfalfa on growth performance and meat and bone quality of broiler genotypes[J]. British Poultry Science, 2018, 59(3):318-329.

2. Mosca F, Zaniboni L, Stella S, et al. Slaughter performance and meat quality of Milanino chickens reared according to a specific free-range program $[\mathrm{J}]$. Poultry Science, 2018, 97(4):1148-1154.

3. Thuy Diep A, Larsen H, Rault J L. Behavioural repertoire of free-range laying hens indoors and outdoors, and in relation to distance from the shed[J]. Australian Veterinary Journal, 2018, 96(4):127131.

4. Yu C, Jiang X, Du H, et al. Effects of free-range broiler production on vegetation characteristic and soil physicochemical property of the stocking areas in China[J]. Agroforestry Systems, 2019(2).

5. Ferreira T C R, Buery J C, Moreira N I B, et al. Toxoplasma gondii: isolation, biological and molecular characterisation of samples from freerange Gallus gallus domesticus from countryside Southeast Brazil[J]. Revista Brasileira De Parasitologia Veterinária, 2018, 27(3):384-389.

6. Sharma N, Hunt P W, Hine B C, et al. Detection of Ascaridia galli, infection in free-range laying hens[J]. Veterinary Parasitology, 2018, 256:9-15.

7. Miguel P E, Valente A H, Thamsborg S M, et al. Antiparasitic activity of chicory (Cichorium intybus) and its natural bioactive compounds in livestock: a review[J]. Parasites \& Vectors, 2018, 11(1):475-488.

8. Sánchez-Gutiérrez RA, Espinoza-Canales A, MuroReyes A, et al. Growth and production of autumnwinter canola (Brassica napus L.) forage in Zacatecas, Mexico[J]. Revista Fitotecnia Mexicana, 2018; 41: 211-216.

9. Chai J, Li Y, Ma X, et al. Experiment on feeding effect of high yield feed rapeseed[J]. Animal Husbandry and Feed Science, 2011, 000(011):19-20. (In Chinese)

10. Chinyelu N I, Karabo S, Chilaka A M. Chemical Composition and Nutritive Benefits of Chicory $\backslash r$, (Cichorium intybus) $\backslash r$, as an Ideal Complementary and/or Alternative Livestock Feed Supplement[J]. Scientific World Journal, 2017:1-11.

11. Tian B, Ran X, Xue H, et al. Gray-scale correlation analysis of nutritional value of 42 wild herbage species in Guizhou Province[J]. Acta Prataculturae Sinic, 2004(1):92-103. (In Chinese)

12. Marley C L, Cook R, Keatinge R, et al. The effect of birdsfoot trefoil (Lotus corniculatus) and chicory
(Cichorium intybus) on parasite intensities and performance of lambs naturally infected with helminth parasites[J]. Veterinary Parasitology, 2003, 112(1-2):147-155.

13. Fuhrmann M, Lasat M M, Ebbs S D, et al. Uptake of Cesium-137 and Strontium-90 from Contaminated Soil by Three Plant Species; Application to Phytoremediation[J]. Journal of Environmental Quality, 2002, 31(3): 904-909.

14. Heuzé V, Tran G, Lebas F. Rape forage. Rape forage. https://www.feedipedia.org/node/15683. 2019. 